\title{
Ocular cicatricial pemphigoid (Review)
}

\author{
DANIEL CONSTANTIN BRANISTEANU ${ }^{1 *}$, GABRIELA STOLERIU $^{2}$, DACIANA ELENA BRANISTEANU ${ }^{3 *}$, \\ DANIEL BODA $^{4}$, CATALINA IOANA BRANISTEANU ${ }^{5}$, MINELA AIDA MARANDUCA ${ }^{6}$, \\ ANDREEA MORARU $^{1 *}$, HORIA TUDOR STANCA $^{7 *}$, MIHAIL ZEMBA $^{7}$ and FLORIAN BALTA ${ }^{7}$ \\ ${ }^{1}$ Department of Ophthalmology, 'Grigore T. Popa' University of Medicine and Pharmacy, 700115 Iasi; \\ ${ }^{2}$ Clinical Department, Faculty of Medicine and Pharmacy, 'Dunărea de Jos' University of Galați, 800008 Galați; \\ ${ }^{3}$ Department of Dermatology, 'Grigore T. Popa' University of Medicine and Pharmacy, 700115 Iasi; \\ ${ }^{4}$ Dermatology Research Laboratory, 'Carol Davila' University of Medicine and Pharmacy, 050474 Bucharest; \\ ${ }^{5}$ 'Grigore T. Popa' University of Medicine and Pharmacy, 700115 Iasi; ${ }^{6}$ Department of Physiology, \\ 'Grigore T. Popa' University of Medicine and Pharmacy, 700115 Iasi; ${ }^{7}$ Department of Ophthalmology, \\ 'Carol Davila' University of Medicine and Pharmacy, 020021 Bucharest, Romania
}

Received March 6, 2020; Accepted April 6, 2020

DOI: $10.3892 /$ etm.2020.8972

\begin{abstract}
Ocular cicatricial pemphigoid is a particular form of mucous membrane pemphigoid and it is characterized by a chronic bilateral conjunctivitis with relapsing-remitting periods. Without therapy $75 \%$ of the cases develop visual loss due to major ocular complications (e.g. severe dry-eye syndrome, corneal erosions, corneal keratinization, entropion, symblepharon). Pathogenesis remains uncertain and probably linked to an autoimmune type II hypersensitivity response in patients with a genetic predisposition and exposure to different environmental triggers. With a worldwide distribution, no racial predilection and an estimated incidence that largely varies from 1/10,000-1/60,000, ocular cicatricial pemphigoid predominantly affects women aged $\sim 60$ years. Conjunctival biopsy with direct immunofluorescence is the gold standard in diagnosis confirmation, but up to $40 \%$ of the patients have a negative biopsy result that does not rule out the diagnosis. The skin and many other mucous membranes (e.g. oral, trachea, esophagus, pharynx, larynx, urethra, vagina and anus) may be involved. The disease grading relies on Foster staging system (based on clinical signs) and Mondino and Brown system (based on the
\end{abstract}

Correspondence to: Professor Mihail Zemba, Department of Ophthalmology, 'Carol Davila' University of Medicine and Pharmacy, 37 Dionisie Lupu Street, District 2, 020021 Bucharest, Romania E-mail:mhlzmb@yahoo.com

Professor Gabriela Stoleriu, Clinical Department, Faculty of Medicine and Pharmacy, 'Dunărea de Jos' University of Galați, 35 Alexandru Ioan Cuza Street, 800008 Galați, Romania

E-mail: stoleriugabriela@yahoo.com

*Contributed equally

Key words: ocular pemphigoid, mucous membrane pemphigoid, cicatricial pemphigoid immunofluorescence, symblepharon, ankyloblepharon inferior fornix depth loss). The differential diagnosis includes atopy, allergies, trauma, chemical burns, radiation, neoplasia, infectious, inflammatory and autoimmune etiologies. The main goals of the treatment are to stop disease progression, to relieve symptoms and to prevent complications. With long-term systemic therapy $90 \%$ of the cases can be efficiently controlled. While Dapsone is the first-line treatment in mild to moderate disease in patients without G6PD deficiency, more severe cases require immunosuppressant therapy with azathioprine, mycophenolate mofetil, methotrexate or cyclosporine. Cyclophosphamide, biologics (etanercept or rituximab) and intravenous immunoglobulin therapy are usually reserved for recalcitrant disease and unsatisfactory results to conventional therapy. Dry eye syndrome requires constant lubricating medication and topical steroids, cyclosporine-A and tacrolimus. Surgery should be planed only in quiescent phase as minor conjunctival trauma can significantly worsen the disease.

\section{Contents}

1. Introduction

2. Pathology

3. Diagnosis and classification

4. Differential diagnosis

5. Treatment

6. Management of complications

\section{Introduction}

Ocular cicatricial pemphigoid (OCP) is classified as a subtype of mucous membrane pemphigoid (MMP) (1). Hallmark of the OCP is the bilateral chronic autoimmune relapsing-remitting conjunctivitis responsible for significant impairment and visual loss due to conjunctival scarring (2). The skin can be involved (in $25 \%$ of patients) and also many other mucous membranes (e.g. oral - estimated in 85-100\% of patients, trachea, esophagus, 
pharynx, larynx, urethra, vagina and anus). Dysphagia was recently reported as a presenting symptom (3). The incidence rates largely vary from 1 per 10,000 to 60,000 (4), with no racial predilection and women twice as affected than men. With no geographical predilection and worldwide distribution, the age of onset is considered to be $\sim 60$ to 80 years (some cases reported at the age of 12-19 years). Systemic practolol therapy (used in cardiac arrhythmias), topical antivirals (e.g. idoxuridine) and antiglaucomatous drugs (e.g. pilocarpine, timolol, epinephrine, parasympathomimetic derivatives, phospholine iodide) have been identified as triggers of OCP.

\section{Pathology}

The precise pathogenesis of the disease remains unclear. There is to date strong evidence for a type II hypersensitivity response involving circulating autoantibodies against different subunits of integrin and laminin that are mainly located in the hemidesmosome-epithelial membrane complex of the conjunctival and squamous epithelium $(5,6)$. The autoantibody-autoantigen conflict activates numerous inflammatory mediators [interleukin-1 (IL-1), IL-13, tumor necrosis factor- $\alpha$ (TNF- $\alpha$ ), migration inhibition factor, macrophage colony-stimulating factor] (7) and proteolytic enzymes responsible for the separation of the epithelium from the basement membrane with subsequent bullae formation (8). Migration of lymphocytes, eosinophils, neutrophils, and mast cells into the substantia propria is responsible for the chronic conjunctivitis manifestations. As inflammation persists, a consistent lymphocytic infiltration appears leading to fibroblast activation, collagen production and scar formation. Mucin deficiency, lacrimal gland and meibomian gland dysfunction leads to severe dry eye syndrome. Also, massive neutrophilic infiltrate of lacrimal gland result in elevated levels of IL-8, matrix metalloproteinase (MMP)-8 and -9 , and myeloperoxidase (MPO) (9-11). Corneal complications include persistent epithelial defects and stromal ulcerations due to entropion and trichiasis and also complete corneal keratinization with neovascularization due to limbal stem cell failure. Symblepharon and ankyloblepharon mutilate the fornixes and also limit the ocular movements.

A genetic predisposition was revealed due to the presence of human leukocyte antigen DR2 (HLA-DR2), DR4 (HLA-DR4 [HLA-DR*0401]), and DQw7 (HLA-DQw7 [DQB1*0301]) genotypes (12).

\section{Diagnosis and classification}

In patients with MMP the ocular involvement is the second most frequent (61\% of the cases) after oral involvement (90\% of the cases). Also, the ocular involvement of MMP is considered high risk and predictor for a poorer prognosis than oral mucosa and/or skin alone involvement (13). Usually the ocular symptoms include red eye, blepharospasm, tearing, photophobia and decreased vision. Burn sensation, foreign body sensation, itching and heavy eyelid are mainly related to the dry eye syndrome. Slit-lamp evaluation can reveal abnormalities of the eyelids (trichiasis, distichiasis, meibomian gland dysfunction and blepharitis), conjunctival hyperemia, papillae and follicles. In more severe cases a keratinization of the conjunctiva is noticed accompanied by subepithelial fibrosis, conjunctival shrinkage and subsequent foreshortening of fornices. Scarring of mucosa in the nose and the mouth is frequent.

Conjunctival biopsy with direct immunofluorescence is the gold standard in OCP diagnosis confirmation. A typical positive result will reveal in the epithelial basement membrane a linear deposition of different immunoglobulins (IgG, Ig A, IgM) and complement 3 proteins. Still, 20-40\% of patients have a negative biopsy result that does not rule out the diagnosis $(14,15)$. Radioimmunoassay and immunoblot techniques can identify the circulating autoantibodies in active disease while classic indirect immunofluorescence is not helpful. The diagnosis is mainly based on ocular clinical findings $(16,17)$. Despite several clinical scoring systems for OCP (Foster, Mondino and Brown), there is no consensus regarding which system clinicians should use. Mondino and Brown Classification System is based on inferior forniceal depth loss (18). Normally, the forniceal depth is $\sim 11 \mathrm{~mm}$. Stage I is defined by a forniceal depth loss up to $25 \%$, stage II by $25-50 \%$, stage III by $50-75 \%$ and stage IV by $>75 \%$ inferior forniceal depth loss. In Foster's Classification System based on clinical findings, progression of disease can be divided into 4 stages (19):

Stage I: characterized by early nonspecific symptoms and minimal findings like chronic mild conjunctivitis, tear dysfunction, and subconjunctival fibrosis. Stage II: cicatrization with conjunctival shrinkage, distorted anatomy and foreshortening of fornices. Stage III: Symblepharon formation, subepithelial scarring, and entropion. Stage IV: Dry eye with ankyloblepharon, extensive adhesions between the eyelid and the globe, resulting in restricted motility; conjunctival and corneal keratinization with persistent epithelial defects, stromal ulceration, and corneal neovascularization.

It is important to mention that untreated disease progresses in up to $75 \%$ of patients despite clinical quiescence, so called 'white inflammation' (20). The proper diagnosis is based on clinical signs and also on positive direct immunofluorescence testing of the conjunctiva and conjunctival biopsy. Serological testing is not of particular interest.

\section{Differential diagnosis}

The differential diagnosis includes atopy, allergies, trauma, chemical burns, radiation and neoplasia. Infectious etiologies (e.g. trachoma), inflammatory etiologies (e.g. rosacea), autoimmune etiologies (e.g. IgA disease) and Graft Versus Host Disease (GVHD) should also be considered (21-27).

Pseudopemphigoid medicamentosa is a common similar clinical finding with drug-related origin. Long-term use of certain topical drugs (pilocarpine, epinephrine, timolol, idoxuridine, echothiophate iodide, and demecarium bromide) $(3,28)$ has been associated with disease onset.

\section{Treatment}

The main goals of the treatment are to stop disease progression, to relieve symptoms and to prevent complications. With long-term systemic therapy $90 \%$ of the cases can be efficiently controlled, and only $10 \%$ of the cases will progress, as compared to $75 \%$ of the cases progressing without treatment. Systemic anti-inflammatory and immunomodulatory medication is 
different according to disease severity and require prompt monitoring due to numerous systemic complications.

In mild disease and in patients without glucose-6-phosphate dehydrogenase (G6PD) deficiency, Dapsone is the first-line agent at a starting dose of $50 \mathrm{mg} /$ day and slowly increased by up to $25 \mathrm{mg}$ every 7 days to the effective dose, between $100-200 \mathrm{mg} / \mathrm{day}$. A careful evaluation during treatment is required as hemolysis and methemoglobinemia can occur. Oral sulfasalazine (1,000-4,000 mg daily) or sulfapyridine (500-1,000 mg daily) might represent an option in cases not suitable for Dapsone (29). If symptoms are not controlled within 3 months, systemic azathioprine or low-dose methotrexate has to be considered, with a special attention for systemic complications such as hepatotoxicity, nephrotoxicity or pancytopenia.

In moderate disease immunomodulatory/immunosuppressive therapy is initiated simultaneously with corticosteroids that allow a quicker and better control of acute phases of severe or rapidly progressive disease. The steroids will be tapered slowly once the disease is under control. In patients without thiopurine methyltransferase (TPMT) deficiency, azathioprine is a valid alternative to treatment despite numerous possible systemic complications such as myelosuppression, infection, malignancy, and drug-induced hypersensitivity syndrome (29). The Systemic Immunosuppressive Therapy for Eye Diseases Cohort Study (SITE), one of the largest retrospective cohort studies of patients with noninfectious ocular inflammatory diseases, showed that cyclophosphamide was effective in $70.7 \%$ of patients with OCP in controlling inflammation at 1 year, with $66.9 \%$ patients on low doses of prednisone ( $\leq 10 \mathrm{mg}$ ) (20). Mycophenolate mofetil has proved effective and well tolerated at a daily dose between 1,000 and 2,000 mg (29). While cyclosporine effectiveness has been reported as variable, tetracyclines are well-tolerated and effective in mild to moderate OCP, especially combined with nicotinamide (30).

In severe disease, orally or intravenously cyclophosphamide is first line of treatment. Adding steroids will allow a more rapid control. A short three-days pulsed intravenously cyclophosphamide therapy can also be used to control the disease in particular conditions, such as rapid progression or prior to surgery. Despite significant systemic complications (myelosuppression, carcinogenesis, and teratogenicity) cyclophosphamide proved effective in SITE-study in controlling inflammation in $80.8 \%$ of patients with OCP at 1 year, with $58.5 \%$ of patients on low doses of prednisone (31). Intravenous Immunoglobulin (IVIG) administration has also been found effective in severe disease, but due to significant systemic complications (anaphylaxis, disseminated intravascular coagulation, acute renal failure) it is reserved for refractory cases. Anti-TNF agents etanercept and infliximab, IL-2 antagonist daclizumab and the anti-CD20 antibody rituximab (alone or in combination with IVIG) also proved efficacy in patients with refractory OCP (32).

Topical therapy is an important adjunct but never a substitute to the systemic treatment. Long-term proper lubrication of the ocular surface can be achieved with preservative-free artificial tears, lubricating ointments and silicone punctal plugs. Advanced formulations restore moisture and also prevent fluid loss. Severe keratitis not improved by tear substitutes or autologous serum drops benefit from long-term administration of topical cyclosporine-A and tacrolimus. Oral doxycycline helps controlling local inflammation. Topical and subconjunctival steroids can be used for short-term only to relieve symptoms. On long-term administration they are responsible for major local complications (glaucoma, cataract). Subconjunctival mitomycin-C injection offered heterogeneous results in small case series (31). Qualitative tear testing has shown promising results in OCP. While membrane array analysis of tear proteins better quantifies the efficacy of systemic immune therapy efficacy when IL-8 and MMP-9 values decrease (9), the MPO levels can be used as specific quantitative marker of disease activity (1,33-35). If proper systemic treatment can control the disease for years allowing a successful discontinuation, the local treatment is usually for life (especially the tear substitutes) requiring periodic evaluation. A recurrence of disease has been noted in approximately one third of the patients (31).

\section{Management of complications}

Surgery of complications is a difficult task as minor conjunctival trauma can worsen the disease. As a general rule, it is recommended the case should be deferred, if possible, until the disease is quiescent. In particular, surgery for entropion, symblepharon or ankyloblepharon carries a higher risk for exacerbation $(36,37)$. Scleral lenses protect the cornea against desiccation and mechanical trauma from trichiasis. Retractor plication technique offers good results in entropion surgery without involving the conjunctiva. Amniotic membrane transplantation and mucous membrane grafting are used to reconstruct the conjunctival fornices. Cryotherapy rather than the mechanical epilation is preferred as treatment of trichiasis. Sutureless amniotic membrane grafting provides growth factors and anti-inflammatory cytokines, similar to autologous serum drops that promote epithelial healing. Cataract surgery has a better prognosis than glaucoma surgery due to minimal invasive technique based on clear corneal small incisions. A keratoprosthesis or osteo-odonto-keratoprosthesis might represent the last chance for maintaining vision in severe end-stage disease with massive corneal keratinization as corneal transplantation has a very poor prognosis in such cases. The use of systemic corticosteroids perioperative can lower the risk of iatrogenic exacerbation.

\section{Acknowledgements}

Not applicable.

\section{Funding}

No funding was received.

\section{Availability of data and materials}

Not applicable.

\section{Authors' contributions}

DCB and DEB contributed to the study design, participated in the entire review process and prepared the manuscript. DB, CIB, AM and HTS contributed to the relevant literature research, and the analysis and critical interpretation of the data. GS, MZ, MAM and FB conceived the study and revised the manuscript. All authors read and approved the final manuscript. 


\section{Ethics approval and consent to participate}

Not applicable.

\section{Patient consent for publication}

Not applicable.

\section{Competing interests}

The authors declare that they have no competing interests.

\section{References}

1. Arafat SN, Suelves AM, Spurr-Michaud S, Chodosh J, Foster CS Dohlman CH and Gipson IK: Neutrophil collagenase, gelatinase, and myeloperoxidase in tears of patients with Stevens-Johnson syndrome and ocular cicatricial pemphigoid. Ophthalmology 121: 79-87, 2014.

2. Robert HR Jr (ed): Ophthalmic Pathology and Intraocular Tumors. In: Basic and Clinical Science Course (BCSC). American Academy of Ophthalmology, pp54-56, 2014.

3. External Disease and Cornea: In: Basic and Clinical Science Course (BCSC). American Academy of Ophthalmology, pp344-345, 2014.

4. Dacosta J: Ocular cicatricial pemphigoid masquerading as chronic conjunctivitis: A case report. Clin Ophthalmol 6: 2093-2095, 2012

5. Chan RY, Bhol K, Tesavibul N, Letko E, Simmons RK, Foster CS and Ahmed AR: The role of antibody to human beta4 integrin in conjunctival basement membrane separation: Possible in vitro model for ocular cicatricial pemphigoid. Invest Ophthalmol Vis Sci 40: 2283-2290, 1999.

6. Tyagi S, Bhol K, Natarajan K, Livir-Rallatos C, Foster CS and Ahmed AR: Ocular cicatricial pemphigoid antigen: Partial sequence and biochemical characterization. Proc Natl Acad Sci USA 93: 14714-14719, 1996.

7. Saw VPJ, Schmidt E, Offiah I, Galatowicz G, Zillikens D, Dart JK, Calder VL and Daniels JT: Profibrotic phenotype of conjunctival fibroblasts from mucous membrane pemphigoid. Am J Pathol 178: 187-197, 2011.

8. Saw VPJ, Offiah I, Dart RJ, Galatowicz G, Dart JK, Daniels JT and Calder VL: Conjunctival interleukin-13 expression in mucous membrane pemphigoid and functional effects of interleukin-13 on conjunctival fibroblasts in vitro. Am J Pathol 175: 2406-2415, 2009.

9. Chan MF, Sack R, Quigley DA, Sathe S, Vijmasi T, Li S, Holsclaw D, Strauss EC and McNamara NA: Membrane array analysis of tear proteins in ocular cicatricial pemphigoid. Optom Vis Sci 88: 1005-1009, 2011.

10. Filip-Ciubotaru F, Manciuc C, Stoleriu G and Foia L: NADPH Oxidase: Structure and activation mecanisms (review). Note I. Rev Med Chir Soc Med Nat Iasi 120: 29-33, 2016.

11. Căruntu C, Boda D, Musat S, Căruntu A and Mandache E: Stress-induced mast cell activation in glabrous and hairy skin. Mediators Inflamm 2014: 105950, 2014.

12. Ahmed AR, Foster S, Zaltas M, Notani G, Awdeh Z, Alper CA and Yunis EJ: Association of DQw7 (DQB1*0301) with ocular cicatricial pemphigoid. Proc Natl Acad Sci USA 88: 11579-11582, 1991.

13. Xu HH, Werth VP, Parisi E and Sollecito TP: Mucous membrane pemphigoid. Dent Clin North Am 57: 611-630, 2013.

14. Labowsky MT, Stinnett SS, Liss J, Daluvoy M, Hall RP III and Shieh C: Clinical implications of direct immunofluorescence findings in patients with ocular mucous membrane pemphigoid. Am J Ophthalmol 183: 48-55, 2017.

15. Hingorani $\mathrm{M}$ and Lightman S: Ocular cicatricial pemphigoid. Curr Opin Allergy Clin Immunol 6: 373-378, 2006.

16. Ilie MA, CaruntuC,Lupu M,Lixandru D, TampaM,Georgescu SR, Bastian A, Constantin C, Neagu M, Zurac SA, et al: Current and future applications of confocal laser scanning microscopy imaging in skin oncology. Oncol Lett 17: 4102-4111, 2019.

17. Ilie MA, Caruntu C, Lixandru D, Tampa M, Georgescu SR Constantin MM, Constantin C, Neagu M, Zurac SA and Boda D In vivo confocal laser scanning microscopy imaging of skin inflammation: Clinical applications and research directions. Exp Ther Med 17: 1004-1011, 2019.

18. Mondino BJ and Brown SI: Ocular cicatricial pemphigoid. Ophthalmology 88: 95-100, 1981.
19. Elder MJ, Bernauer W, Leonard J and Dart JK: Progression of disease in ocular cicatricial pemphigoid. Br J Ophthalmol 80: 292-296, 1996.

20. Williams GP, Radford C, Nightingale P, Dart JK and Rauz S: Evaluation of early and late presentation of patients with ocular mucous membrane pemphigoid to two major tertiary referral hospitals in the United Kingdom. Eye (Lond) 25: 1207-1218, 2011.

21. Brănișteanu DE, Pintilie A, Andres LE, Dimitriu A, Oanţă A, Stoleriu G and Brănişteanu DC: Ethiopatogenic hypotheses in lichen planus. Rev Med Chir Soc Med Nat Iasi 120: 760-767, 2016.

22. Schaas BA, Ivan S, Titianu M, Condratovici CP, Maier A and Schaas CM: Biochemical markers predicting the risk of gestational diabetes mellitus. Mater Plast 54: 133-136, 2017.

23. Brănişteanu DE, Brănişteanu DC, Stoleriu G, Ferariu D, Voicu CM, Stoica LE, Căruntu C, Boda D, Filip-Ciubotaru FM, Dimitriu A, et al: Histopathological and clinical traps in lichen sclerosus: A case report. Rom J Morphol Embryol 57 (Suppl 2): 817-823, 2016.

24. Tatu AL, Ionescu MA and Nwabudike LC: Contact allergy to topical mometasone furoate confirmed by rechallenge and patch test. Am J Ther 25: e497-e498, 2018.

25. Branisteanu DE, Pintilie A, Dimitriu A, Cerbu A, Ciobanu D, Oanta A and Tatu AL: Clinical, laboratory and therapeutic profile of lichen planus. Rev Med Chir Soc Med Nat Iasi 125: 25-32, 2017

26. Brănişteanu DE, Cotrutz CE, Luca MC, Molodoi DA, Stoica LE, Ianoşi SL, Cianga CM and Brănişteanu DC: Morphopathological stigmata in acne fulminans. Rom J Morphol Embryol 56: $1185-1190,2015$

27. Tatu AL and Cristea VC: Unilateral blepharitis with fine follicular scaling. J Cutan Med Surg 21: 442, 2017.

28. Tatu AL, Elisei AM, Chioncel V, Miulescu M and Nwabudike LC: Immunologic adverse reactions of $\beta$-blockers and the skin (Review). Exp Ther Med 18: 955-959, 2019.

29. Neff AG, Turner M and Mutasim DF: Treatment strategies in mucous membrane pemphigoid. Ther Clin Risk Manag 4: $617-626,2008$

30. Kempen JH, Daniel E, Gangaputra S, Dreger K, Jabs DA, Kaçmaz RO, Pujari SS, Anzaar F, Foster CS, Helzlsouer KJ, et al: Methods for identifying long-term adverse effects of treatment in patients with eye diseases: The Systemic Immunosuppressive Therapy for Eye Diseases (SITE) Cohort Study. Ophthalmic Epidemiol 15: 47-55, 2008.

31. Pujari SS, Kempen JH, Newcomb CW, Gangaputra S, Daniel E, Suhler EB, Thorne JE, Jabs DA, Levy-Clarke GA, Nussenblatt RB, et al: Cyclophosphamide for ocular inflammatory diseases. Ophthalmology 117: 356-365, 2010.

32. Foster CS, Chang PY and Ahmed AR: Combination of rituximab and intravenous immunoglobulin for recalcitrant ocular cicatricial pemphigoid: A preliminary report. Ophthalmology 117: 861-869, 2010.

33. Ilie MA, Caruntu C, Tampa M, Georgescu SR, Matei C, Negrei C, Ion RM, Constantin C, Neagu M and Boda D: Capsaicin: Physicochemical properties, cutaneous reactions and potential applications in painful and inflammatory conditions. Exp Ther Med 18: 916-925, 2019.

34. Ghiţă MA, Căruntu C, Rosca AE, Căruntu A, Moraru L, Constantin C, Neagu M and Boda D: Real-time investigation of skin blood flow changes induced by topical capsaicin. Acta Dermatovenerol Croat 25: 223-227, 2017.

35. Branisteanu D, Caruntu C, Negrei C, Ghita MA, Caruntu A, Badarau AI, Buraga I, Boda D and Albu A: Capsaicin, a hot topic in skin pharmacology and physiology. Farmacia 63: 487-491, 2015.

36. Lupu MN, Miulescu M, Ștefanopol IA, Stoleriu G, Matei MN, Manolache N, Vasincu D, Ćiobotaru OR and Costuleanu M: Effect of 2,6-diisopropylphenol and 1,1,1,3,3,3-hexafluoro-2-(fluoromethoxy) propane as anesthetic. Rev Chim (Bucharest) 70: 1888-1892, 2019.

37. Ciobotaru OR, Lupu MN, Rebegea LF, Ciobotaru OC, Duca OM, Tatu AL, Voinescu CD, Stoleriu G, Earar K and Miulescu M: Dexamethasone-chemical structure and mechanisms of action in prophylaxis of postoperative side effects. Rev Chim (Bucharest) 70: 843-847, 2019.

This work is licensed under a Creative Commons Attribution-NonCommercial-NoDerivatives 4.0 International (CC BY-NC-ND 4.0) License. 